\title{
OPEN
}

Published online: 10 March 2020

\section{Author Correction: Influence of the magnetic field on bandgap and chemical composition of zinc thin films prepared by sparking discharge process}

\author{
Stefan Ručman (D, Panich Intra, E. Kantarak, W. Sroila, T. Kumpika, J. Jakmunee, \\ W. Punyodom, Biljana Arsić \& Pisith Singjai
}

Correction to: Scientific Reports https://doi.org/10.1038/s41598-020-58183-4, published online 29 January 2020

The original version of this Article contained a repeated typographical error where "Differential Mobility Analyzer" and "Differential Mobility Analysis" were given as "dynamic mobility analyzer" and "dynamic mobility analysis" respectively.

In the Abstract,

"Dynamic mobility analysis (DMA) indicates smaller particles are fabricated by discharging zinc wires in a higher magnetic field."

now reads:

"Differential Mobility Analysis (DMA) indicates smaller particles are fabricated by discharging zinc wires in a higher magnetic field."

In the Results section,

"To investigate nanoparticle distribution of aerosols created by sparking discharge and influence of magnetic field on median aerodynamic diameter we employed dynamic mobility analyzer (DMA), measured at different applied voltage and with and without presence of magnetic field."

now reads:

“To investigate nanoparticle distribution of aerosols created by sparking discharge and influence of magnetic field on median aerodynamic diameter we employed Differential Mobility Analyzer (DMA), measured at different applied voltage and with and without presence of magnetic field.'

In the Methods section,

"In Fig. 7 schematically is represented experimental setup of sparking machine connected to Dynamic Mobility Analyzer (DMA), operating principle was explained previously ${ }^{36,37}$.,

now reads:

"In Fig. 7 schematically is represented experimental setup of sparking machine connected to Differential Mobility Analyzer (DMA), operating principle was explained previously ${ }^{36,37}$." 
In the legend of Table 1, where,

"Table 1. Comparison of results obtained from dynamic mobility analyzer (DMA) by sparking Zinc wire at different conditions. Geometric mean as average particle size, concentration in number of particles per $\mathrm{cm}^{3}$."

now reads:

“Table 1. Comparison of results obtained from Differential Mobility Analyzer (DMA) by sparking Zinc wire at different conditions. Geometric mean as average particle size, concentration in number of particles per $\mathrm{cm}^{3}$."

Additionally, the first two sentences in the Particle distribution (DMA) section are a duplication of the legend of Table 1. The text,

"Table 1. Comparison of results obtained from dynamic mobility analyzer (DMA) by sparking Zinc wire at different conditions. Geometric mean as average particle size, concentration in number of particles per $\mathrm{cm}^{3}$., has been removed from this section.

These errors have now been corrected in the PDF and HTML versions of the Article.

(c) (i) Open Access This article is licensed under a Creative Commons Attribution 4.0 International License, which permits use, sharing, adaptation, distribution and reproduction in any medium or format, as long as you give appropriate credit to the original author(s) and the source, provide a link to the Creative Commons license, and indicate if changes were made. The images or other third party material in this article are included in the article's Creative Commons license, unless indicated otherwise in a credit line to the material. If material is not included in the article's Creative Commons license and your intended use is not permitted by statutory regulation or exceeds the permitted use, you will need to obtain permission directly from the copyright holder. To view a copy of this license, visit http://creativecommons.org/licenses/by/4.0/.

(c) The Author(s) 2020 